\section{Fast Construction of Force-Closure Grasps}

\author{
Chao-Ping Tung and Avinash C. Kak
}

\begin{abstract}
This article presents a new theorem and an algorithm for fast synthesis of two-fingered force-closure grasps for arbitrary polygonal objects. The polygonal objects are allowed to be of arbitrary shape, in the sense that there is no restriction that the objects be convex. Moreover, each edge of the polygon is allowed to have different frictional characteristics. Our novel formulation results in a simple and efficient algorithm that finds grasps that tolerate the largest positioning errors for each of the fingertips at the contact points. The algorithm is both complete and correct, meaning that if any force-closure grasp exists for the object, the algorithm will find it; furthermore, all the grasps synthesized by the algorithm are guaranteed to be valid force-closure grasps.
\end{abstract}

\section{INTRODUCTION}

Grasp determination, the automatic generation of grasp configurations for a given object and a given gripper, is an important branch of robotics; the subject has therefore attracted much attention over the last decade. While much of what was published early dealt with the parallel-jawed grippers [1]-[7], the more recent work has focussed on dextrous grippers with multiple fingers [8]-[17]. The parallel-jaw work in the published literature has dealt mostly with the determination of grasps that would allow a robot to retrieve or place an object in an environment containing obstacles [1], [2]; with the determination of a grasp given the need to balance all the frictional and gravitational forces [4], [5]; with optimum strategies for the selection of placement points for the two jaws [3]; with the planning of a grasp motion such that the object in its final grasped pose would occupy a designated orientation [6]; and so on. These parallel-jaw contributions have not addressed explicitly the issue of how to synthesize a force-closure grasp.

The issue of force closure first arose in the context of dextrous grasps. A grasp is considered to be of force-closure type if it permits the robot to counteract any disturbance force or torque applied to the grasped object. In other words, the finger placements for a forceclosure grasp have to be such that by altering the directions and/or the magnitudes of the applied contact forces, the robot is able to keep the object grasped despite any externally applied forces/torques that might otherwise cause the object to slide or rotate. In the more modern literature on grasping, the concepts of force closure have been expounded on by Li and Sastry [16], Nguyen [15], Park and Starr [12], Mishra, Schwartz, and Sharir [8], Faverjon and Ponce [13], Markenscoff, Ni, and Papadimitriou [9], Hong, Lafferriere, Mishra and Tan [10], Ferrari and Canny [14], Kirkpatrick, Mishra, and Yap [17], and others.

The previous work that is most relevant to our contribution here is that of Nguyen [15] where the author presented a geometrical algorithm for computing the force-closure grasps for polyhedral objects. The algorithm identifies the regions of the different faces of a polyhedral object where the placement of the fingers would guarantee force closure. It is not too difficult to illustrate the basic idea used in [15] for the case of polygonal objects. Consider the polygonal object $A B C D$ represented by the shaded portion in Fig. 1(a). Assume that

Manuscript received August 4, 1994; revised August 1, 1995. This paper was recommended for publication by Associate Editor V. Kumar and Editor A Desrochers upon evaluation of reviewers' comments. This work was supported by Nippondenso Co., Ltd.

The authors are with the School of Electrical Engineering, Purdue University, West Lafayette, IN 47907-1285 USA

Publisher Item Identifier S 1042-296X(96)03833-5.

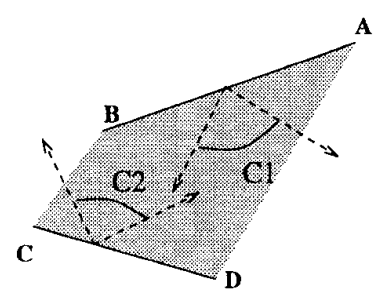

(a)

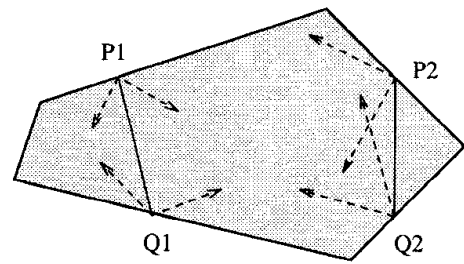

(b)

Fig. 1. Friction cones at the contact points for three grasps. (a) Friction cones of $\overline{\mathrm{AB}}$ and $\overline{\mathrm{CD}}$. (b) Two grasps for an object.

the friction cones for the edges $\mathrm{AB}$ and $\mathrm{CD}$ are as illustrated there. The principles of mechanics dictate that if the goal is to grasp the object by making contacts with the surfaces $A B$ and $C D$, the forces at the two contacts must be such that they point not only into their respective friction cones but, also, each force must point into the friction cone corresponding to the other surface. For example, for the two grasps depicted in Fig. 1(b), the grasp with contact points $\left\{P_{2}, Q_{2}\right\}$ is not a force-closure grasp, since there do not exist usable force directions at $P_{2}$ and $Q_{2}$-recall that the applied forces at each of these points must point into their respective friction cones and that the force at $P_{2}$ would point into the friction cone at $Q_{2}$ and vice versa. By contrast, there does exist a grasp with contact points $\left\{P_{1}, Q_{1}\right\}$ that is of force-closure type.

Nguyen formalized this criterion for selecting grasp points by using a double-sided cone formed by the two friction cones, one for each edge. As shown in Fig. 2(b), this double-sided cone is formed by the intersection of the angle $\mathrm{C} 1$, which corresponds to the friction cone for side $\mathrm{AB}$, and the angle $-\mathrm{C} 2$, which corresponds to a mirror reflection of the friction cone of angle $C_{2}$ for side $\mathrm{CD}$. We will refer to such a double-sided cone as a grasp generating cone (GGC).

Nguyen used the fact that in order to locate two contact points for a force-closure grasp, all we have to do is to situate the grasp generating cone somewhere within the object and use for finger placement any of the points within the segments created by the intersection of the grasp generating cone with the sides $A B$ and $C D$. While the reasoning for selecting, say, two contact points is as simple as what we have presented here, Nguyen went a step further and presented an algorithm for locating the grasp generating cone in such a manner as to yield the longest possible segments on the surfaces $A B$ and $\mathrm{CD}$ in our example. This algorithm basically constructs a triangle for each object edge; this triangle would be generated if we scanned the entire space by the grasp generating cone and if we insisted that the bounding edges of this cone always intersect the two object edges. Shown in Fig. 2(c) are the two triangles, one for $A B$ and the other for $\mathrm{CD}$. Nguyen then claimed that the optimum placement of the grasp generating cone was such that its apex lay within the intersection region of the triangles and presented an algorithm for finding this 


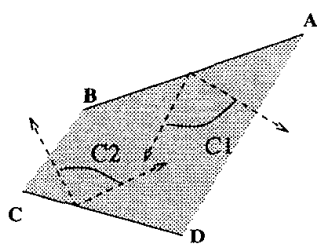

(a)

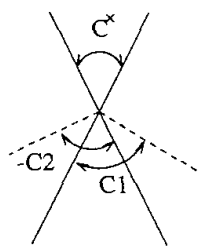

(b)

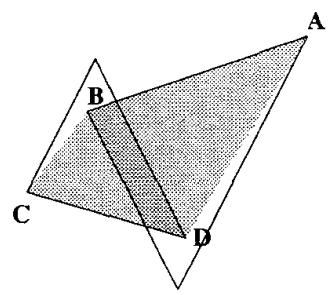

(c)

Fig. 2. Some steps of Nguyen's algorithm. (a) Friction cones of $\overline{\mathrm{AB}}$ and $\overline{\mathrm{CD}}$. (b) Grasp generating cone for $\overline{\mathrm{AB}}$ and $\overline{\mathrm{CD}}$. (c) Triangles for $\overline{\mathrm{AB}}$ and $\overline{\mathrm{CD}}$.

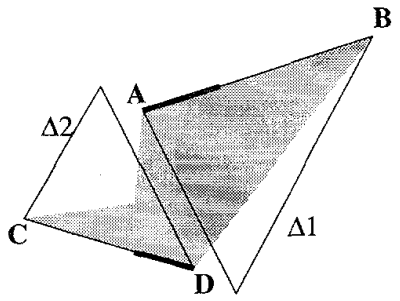

Fig. 3. Certain object shapes, such as the one represented by the shaded region here, cannot be handled by the grasp synthesis algorithm of [15]. $\Delta 1$ and $\Delta 2$ are the triangles generated by Nguyen's algorithm. Since $\Delta 1 \cap \Delta 2=\emptyset$, his algorithm cannot be used. The high-lighted portions of the edges labeled $\mathrm{AB}$ and $\mathrm{CD}$ are the regions of an optimum grasp discovered by our algorithm for this object

optimum placement. Subsequently, he generalized the algorithm to the case of 3-D polyhedral objects.

Although a pioneering contribution for its time, we have found the algorithm presented in [15] to be slow and cumbersome, not to speak of the fact that it cannot be used when the different surfaces have different frictional characteristics. Also, we discovered that Nguyen's algorithm is incapable of handling certain kinds of polygonal shapes. Consider, for example, the object represented by the shaded region in Fig. 3. The two triangles in this case for the object edges $A B$ and $\mathrm{CD}$ are such that they have zero intersection region, implying that Nguyen's algorithm cannot be used here. However, our algorithm discovered an optimum grasp shown by the highlighted portions of the edges $\mathrm{AB}$ and $\mathrm{CD}$.

In the ensuing discussion, we present a simple and fast analytical algorithm for generating force-closure grasps for polygonal objects of arbitrary shape and arbitrary composition. By arbitrary composition, we mean the edges are allowed to possess different frictional characteristics. We model the contact between a finger and an edge as a point contact with friction. This model is a conservative estimate for fingers made from soft materials, since a soft finger contacting an edge can be viewed as an edge contact with friction, which is equivalent to two point contacts with friction, one at each end of the region of contact. We then extend this algorithm to the case of 3-D polyhedral objects.

\section{SOME REPRESENTATIONAL IsSues}

In Section V, we will present a new theorem and subsequently a new algorithm for synthesizing optimal grasps for polygonal objects.

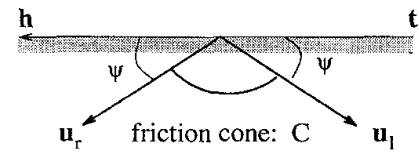

Fig. 4. Characterizing the friction cone associated with an object edge.

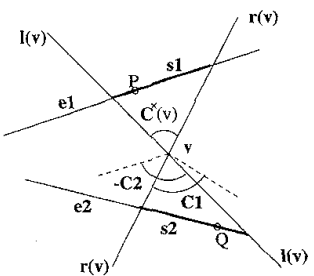

Fig. 5. The grasp with contacts at $P$ and $Q$ is a force-closure grasp

That theorem and the algorithm utilize a specific representation for any pair of edges for which a force-closure grasp is desired. Explication of this representation is the major focus of this section. Other representational considerations included in this section deal with the notation for friction cones, notation for the grasp generating cone, and the representation used for the independent regions of contact that are obtained by intersecting a given pair of edges with a grasp generating cone.

\section{A. Representation of Friction Cones}

The friction cone associated with an object edge is characterized by the smallest angle, denoted $\psi$, between the edge and the boundaries of the friction cone; these boundaries, in turn, are represented by two unit direction vectors, $\mathbf{u}_{l}$ and $\mathbf{u}_{r}$, which are parallel to the boundaries of the friction cone to one's left and right, respectively, if one stands on the edge facing the material side. A pictorial illustration of these terms is given in Fig. 4.

\section{B. Representation of a Grasp Generating Cone}

A grasp generating cone is characterized by the location of its central vertex and by the two lines that correspond to its two defining edges. Therefore, a grasp generating cone, denoted $C^{\times}(\mathrm{v})$, will be represented by the triple:

$$
C^{\times}(\mathbf{v})=(\mathbf{v}, \mathbf{l}, \mathbf{r})
$$

where, as shown in Fig. 5, $\mathrm{v}$ is the location of the central vertex of the cone, $\mathbf{l}(\mathbf{v})$, and $\mathbf{r}(\mathbf{v})$ are the equations of the edges of $C^{\times}(\mathbf{v})$ that are on the left and right, respectively, when one is standing at $v$ and looking outward toward the mouth of $C^{\times}(v)$ in either direction. In our analysis, the equations $\mathbf{l}(\mathbf{v})$ and $\mathbf{r}(\mathbf{v})$ will be expressed in parametric form.

Here is a formal definition of a grasp generating cone [15].

Definition 1: Let $C_{1}$ and $C_{2}$ be the convexes that characterize the friction cones of edges $e_{1}$ and $e_{2}$, respectively, of a polygon $\mathcal{P}$. For a given point $\mathbf{v}$ in the plane containing $\mathcal{P}, C^{\times}(\mathbf{v})$ can be constructed from $C_{1}$ and $C_{2}$ as follows.

1) Translate the convexes $C_{1}$ and $-C_{2}$ so that both of their vertices are located at $\mathrm{v}$.

2) Compute $-C_{2} \cap C_{1}$ to obtain the convex $C$.

3) If $C=\emptyset$, then $C^{\times}(\mathbf{v})=\emptyset$. Otherwise, $C^{\times}(\mathbf{v})$ is the doublesided cone obtained by extending the boundaries of the convex C.

\section{Representation of Edge Pairs for Grasp Analysis}

The representation that we will present in the present section for edge pairs may be thought of as a procedural representation, in the sense that each pair of edges is converted into this representation on 


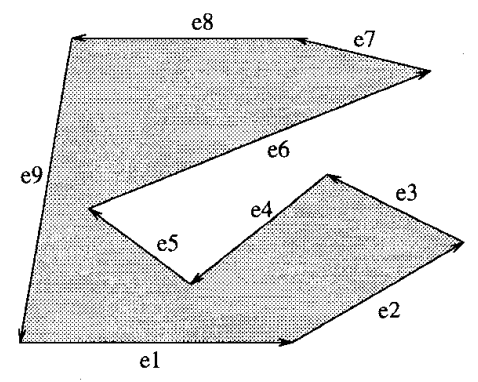

Fig. 6. The boundary of a polygon.

the fly as the entire object is analyzed for different possible grasps. Prior to analysis for the discovery of force-closure grasps, the object is stored in the long-term memory as sequences of directed edges, the sequences corresponding to the outermost contour of the object and any holes that the object might contain. In each sequence, the edge directions are chosen such that the material side of the object is always on the left. Each edge $e_{i}$ in each sequence is represented by the triple $\left(\mathbf{t}_{i}, \mathbf{u}_{i}, d_{i}\right)$ needed for the parametric form

$$
e_{i}=\mathbf{t}_{i}+\alpha_{i} \mathbf{u}_{i}, \quad 0 \leq \alpha_{i} \leq d_{i}
$$

where the tail vector $\mathbf{t}_{i}$, the unit vector $\mathbf{u}_{i}$ and the parameter $\alpha_{i}$, ranging over the interval $\left[0, d_{i}\right]$, have obvious meanings. To recapitulate a familiar property of these edges that is important to us, note that if we picked any two distinct edges, for some pairs of edges the cross-product of their respective unit vectors will be positive, for others it will be negative, and for yet others zero. For the object of Fig. 6, for example,

$$
\mathbf{u}_{1} \times \mathbf{u}_{2}>0, \quad \mathbf{u}_{1} \times \mathbf{u}_{4}<0, \quad \mathbf{u}_{1} \times \mathbf{u}_{8}=0
$$

where the $\times$ operator denotes a 2-D cross-product ${ }^{1}$, i.e., $\left(x_{i}, y_{i}\right)^{T} \times$ $\left(x_{j}, y_{j}\right)^{T}=x_{i} y_{j}-y_{i} x_{j}$.

Given an object representation as discussed above, synthesis of force-closure grasps consists of picking any two edges and transforming them into what we will refer to as the standard configuration. As the reader shall see, this transformation of representation, although seemingly superfluous at first sight, will permit application of the same grasp synthesis algorithm to every pair of edges, no matter what the orientation of the individual edges in the pair and regardless of whether both edges belong to the same sequence in the object representation or to two different sequences. When two edges come from two different sequences in the object representation, at least one edge is on a hole boundary.

Given any two edges of a polygon, the point of intersection of the lines containing the edges is either external to the edges, as shown in Fig. 7(a), or internal to one of the edges, as in Fig. 7(b). We denote the intersection point with the symbol $\mathbf{O}$. If the intersection point is external to both edges, then we assign special labels, c for "close" and $\mathbf{f}$ for "far," to the vertices of the two object edges, both labels with respect to the point of intersection. The vertex labels for the two edges in Fig. 7(a) are in accord with this convention.

When the lines containing the two edges intersect as shown in Fig. 7(b), the assignment of the "close" and "far" labels to edge vertices becomes slightly less obvious, at least for the edge that

\footnotetext{
1 Since we are dealing with planar objects, we may assume that the objects lie on the xy-plane in the 3-D Euclidean space. Therefore, the direction vectors of the edges can be expressed as $x \mathbf{i}+y \mathbf{j}$. Furthermore, the cross product of any two direction vectors, $\mathbf{u}_{i}$ and $\mathbf{u}_{j}$, is equal to $\left(x_{i} y_{j}-y_{i} x_{j}\right) \mathbf{k}$, which is a vector that lies on the $\mathrm{z}$-axis. Thus, for all practical purposes, we may consider these cross products as being scalars, which we define here to be the results of 2-D cross-products.
}

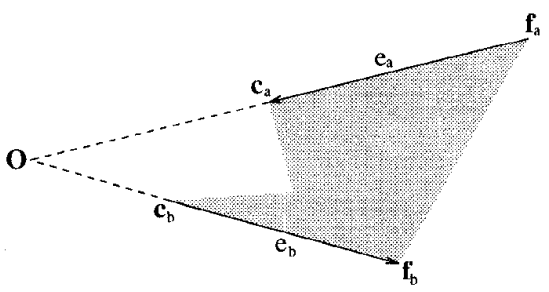

(a)

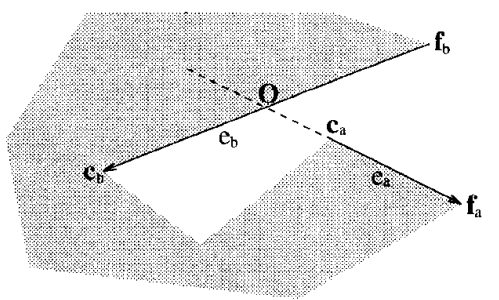

(b)

Fig. 7. Labeling the end points for two pairs of edges. (a) Intersection external to the edges. (b) Intersection internal to $e_{b}$.

contains the intersection point. Now, the edge that does not contain the point of intersection is assigned the vertex labels $\mathbf{c}$ and $\mathbf{f}$ in the same manner as described above. Shown in Fig. 7(b) are the vertex labels $\mathbf{c}_{a}$ and $\mathbf{f}_{a}$ for edge $e_{a}$. The $\mathbf{f}$ and $\mathbf{c}$ labels to the other edge must be such that a traversal from $\mathbf{f}$ to $\mathbf{c}$ yields a material side that is opposite to what it is for the other edge. In other words, if for a traversal from $\mathbf{f}_{a}$ to $\mathbf{c}_{a}$, the material side is on the left, then for the traversal from $\mathbf{f}_{b}$ to $\mathbf{c}_{b}$ the material side must be on the right. The vertex labels $\mathbf{f}_{b}$ and $\mathbf{c}_{b}$ in Fig. 7(b) have been assigned on this basis.

A formal definition of standard configuration is as follows.

Definition 2-Standard Configuration: In the standard configuration, a pair of given edges will be assigned the distinguished labels $e_{P}$ and $e_{Q}$. These labels are assigned in such a manner that the following two conditions, one on the cross-product and the other on the parameterizations, are satisfied.

1) (Cross-product condition:)

$$
\mathbf{u}_{P} \times \mathbf{u}_{Q} \geq 0
$$

where the unit vectors $\mathbf{u}_{P}$ and $\mathbf{u}_{Q}$ are given by $\mathbf{u}_{P}=$ $\left|\mathbf{c}_{P}-\mathbf{f}_{P}\right|$ and $\mathbf{u}_{Q}=\left|\mathbf{f}_{Q}-\mathbf{c}_{Q}\right|$.

2) (Parameterization condition:)

$$
\begin{aligned}
& e_{P}=\mathbf{f}_{P}+\alpha_{i} \mathbf{u}_{P}, \quad 0 \leq \alpha_{i} \leq d_{P}, \\
& e_{Q}=\mathbf{c}_{Q}+\beta_{i} \mathbf{u}_{Q}, \quad 0 \leq \beta_{i} \leq d_{Q} .
\end{aligned}
$$

For example, consider the standard configurations for two pairs of edges of the object shown in Fig. 6. First, consider the edge pair $e_{6}$ and $e_{8}$. Because their cross-product $e_{6} \times e_{8}$ is positive, we set $e_{P} \equiv e_{6}$ and $e_{Q} \equiv e_{8}$. The parameterizations for $e_{6}$ and $e_{8}$ correspond to the arrows shown on the two edges in Fig. 8(a). The parameterizations on $e_{P}$ and $e_{Q}$ remain the same, as shown by the arrows in Fig. 8(b). However, the situation involving the edges $e_{3}$ and $e_{6}$ is different. Since the cross-product $e_{3} \times e_{6}$ is negative, we set $e_{P} \equiv e_{6}$ and $e_{Q} \equiv e_{3}$. The original parameterizations for $e_{3}$ and $e_{6}$ are shown in Fig. 8(c), and the new parameterizations for the edges renamed as $e_{P}$ and $e_{Q}$ in Fig. 8(d). 


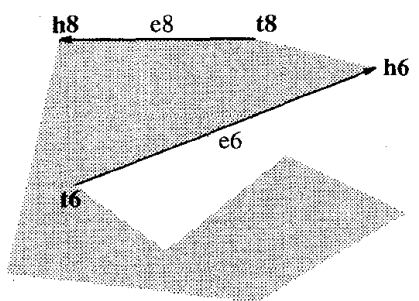

(a)

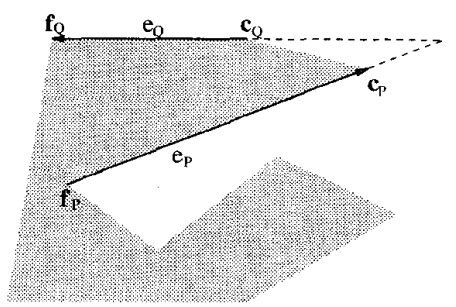

(b)

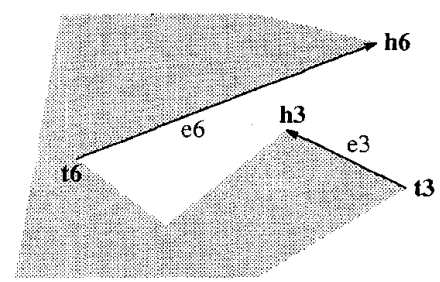

(c)

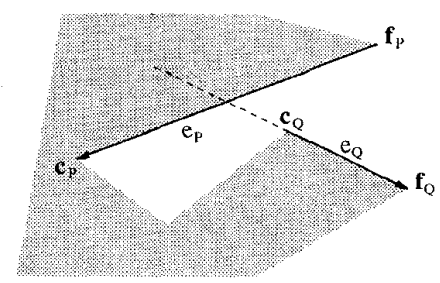

(d)

Fig. 8. The standard configurations for two pair of edges. (a) Parameterizations of $e_{6}$ and $e_{8}$. (b) Standard configuration of $e_{6}$ and $e_{8}$. (c) Parameterizations of $e_{3}$ and $e_{6}$. (d) Standard configuration of $e_{3}$ and $e_{6}$.

\section{Representation of Independent Regions of Contact}

Given two edges that have been assigned the distinguished labels $e_{P}$ and $e_{Q}$, the independent regions of contact on the edges will be denoted $s_{P}$ and $s_{Q}$, respectively. As mentioned in the introduction to this section, the independent regions of contact are obtained by intersecting the edges $e_{P}$ and $e_{Q}$ with the grasp generating cone $C^{\times}(\mathbf{v})=(\mathbf{v}, \mathbf{l}, \mathbf{r})$. In terms of the parameterizations for $e_{P}$ and $e_{Q}$, the points where the GGC intersects the lines containing these two edges will be specified by the points $\left\{\alpha_{r}, \alpha_{l}\right\}$ and $\left\{\beta_{r}, \beta_{l}\right\}$, respectively. Note that the subscripts $l$ and $r$ indicate an intersection with the lines 1 and $\mathbf{r}$, respectively, that characterize the grasp generating cone. In keeping with the parameterizations for two edges in standard configuration, both $\alpha_{r}$ and $\alpha_{l}, \alpha_{r}<\alpha_{l}$, are measured from the end $\mathbf{f}_{P}$. Similarly, both $\beta_{r}$ and $\beta_{l}, \beta_{r}<\beta_{l}$, are measured from the end $c_{Q}$.

For example, consider the situation shown in Fig. 9. The highlighted portions of $e_{P}$ and $e_{Q}$ are the independent regions of contact

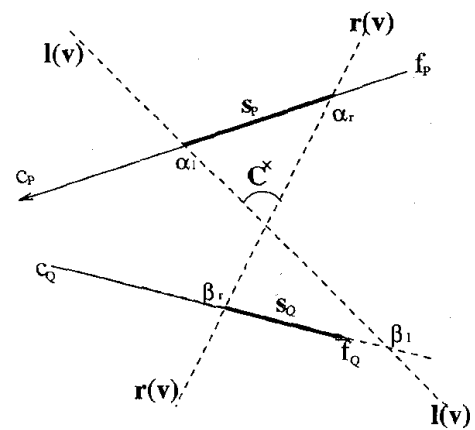

Fig. 9. Parameters for $s_{P}$ and $s_{Q}$.

generated by the grasp generating cone $C^{\times}(\mathbf{v})$. The intersection points of $C^{\times}(\mathrm{v})$ and the line containing $e_{P}$ all lie on $e_{P}$, so the independent region of contact for $e_{P}$ is characterized by $\left\{\alpha_{r}, \alpha_{l}\right\}$, i.e., the end points of $s_{P}$ are $\left(\mathbf{f}_{P}+\alpha_{r} \mathbf{u}_{P}, \mathbf{f}_{P}+\alpha_{l} \mathbf{u}_{P}\right)$. However, the situation for $e_{Q}$ is different. Although the intersection of the line containing $e_{Q}$ and $\mathbf{r}(\mathbf{v})$ lies on $e_{Q}$, the intersection of the line containing $e_{Q}$ and $\mathbf{l}(\mathbf{v})$ does not, i.e., $\beta_{l}>d_{Q}$, where $d_{Q}$ is the length of the edge labeled $e_{Q}$. For this case the independent region of contact $s_{Q}$ is parameterized by $\left\{\beta_{r}, d_{Q}\right\}$. In general, the independent regions of contact $s P$ and $s_{Q}$ are parameterized by $\left\{\operatorname{MAX}\left\{0, \alpha_{r}\right\}\right.$, $\left.\operatorname{MIN}\left\{d_{P}, \alpha_{l}\right\}\right\}$ and $\left\{\operatorname{MAX}\left\{0, \beta_{r}\right\}, \operatorname{MIN}\left\{d_{Q}, \beta_{l}\right\}\right\}$, respectively.

\section{Force Closure in 2-D}

Our introductory discussion on force-closure in Section I implies that a grasp with contact points $P$ and $Q$ on edges $e_{1}$ and $e_{2}$, respectively, is a force-closure grasp if and only if there exists a grasp generating cone $C^{\times}(\mathbf{v})$ such that $P \in s_{1}$ and $Q \in s_{2}$, where $s_{1}$ and $s_{2}$ are the portions of $e_{1}$ and $e_{2}$, respectively, that lie strictly inside of $C^{\times}(\mathrm{v})$. More formally, a grasp has the force-closure property if it satisfies the following two necessary conditions, which we refer to as the Necessary Criteria for Force Closure. The reader should note that these conditions will be stated not using the distinguished labels $e_{P}$ and $e_{Q}$ for the edges. That is because the two edges are first checked for whether or not they satisfy these conditions before the distinguished labels are assigned to them.

1) [Necessary Criterion 1:] The intersection of the friction cone for one edge with the mirror reflection of the friction cone for the other edge cannot be empty for a force-closure grasp to exist. This condition translates into the following constraint on the orientation of the edges:

$$
|\theta|>\psi_{1}+\psi_{2}
$$

where $\theta=\cos ^{-1}\left(\mathbf{u}_{1} \cdot \mathbf{u}_{2}\right)$ and $\psi_{i}$ is the magnitude of the angle between edge $i$ and its friction cone. The unit vectors $\mathbf{u}_{1}$ and $\mathbf{u}_{2}$ correspond to the parameterizations for the two edges in the definition of the object, in accordance with the object representation described at the beginning of Section II-C.

2) [Necessary Criterion 2:] Suppose we designate the material side of an edge as its "front" side. Then the existence of a force-closure grasp for $e_{1}$ and $e_{2}$ implies that the segment $\overline{P Q}$ must be either in front of both edges or behind both edges, as depicted in Fig. 10. To check that this condition is satisfied, we compute the intersection of the lines containing $e_{1}$ and $e_{2}$ :

$$
\begin{aligned}
& \mathbf{t}_{1}+\alpha \mathbf{u}_{1}=\mathbf{t}_{2}+\beta \mathbf{u}_{2} \\
& \Rightarrow\left\{\begin{array}{l}
\alpha=\frac{\left(\mathbf{t}_{2}-\mathbf{t}_{1}\right) \times \mathbf{u}_{2}}{\mathbf{u}_{1} \times \mathbf{u}_{2}} \\
\beta=\frac{\left(\mathbf{t}_{2}-\mathbf{t}_{1}\right) \times \mathbf{u}_{1}}{\mathbf{u}_{1} \times \mathbf{u}_{2}} .
\end{array}\right.
\end{aligned}
$$




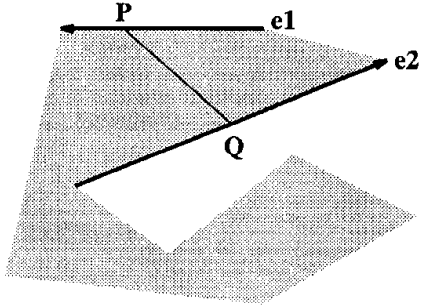

(a)

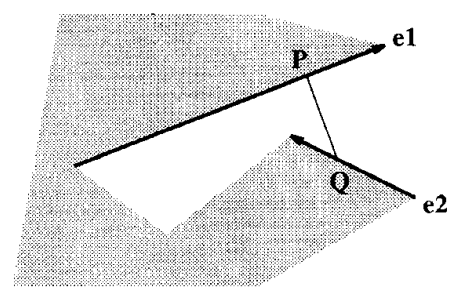

(b)

Fig. 10. Orientations of two edges that satisfy Necessary Criterion 2. (a) $\overline{\mathrm{PQ}}$ is in front of both edges. (b) $\overline{\mathrm{PQ}}$ is behind both edges.

If either $\{\alpha \leq 0 \& \beta \leq 0\}$ or $\left\{\alpha \geq d\left(e_{1}\right) \& \beta \geq d\left(e_{2}\right)\right\}$, then Criterion 2 is violated.

\section{Optimal Force-Closure Grasps}

As mentioned in Section II-D, the intersections of a grasp generating cone $C^{\times}(\mathbf{v})$ with the two edges for which a force-closure grasp is desired are referred to as the independent regions of contact. The grasp with the contact points located in the middle of $s_{1}$ and $s_{2}$ is said to be defined by $s_{1}$ and $s_{2}$. For finding the best forceclosure grasp, one would want these regions to be as wide as possible. Wider regions would be more tolerant to errors in the placement of the fingers. Therefore, a grasp that maximizes the widths of the independent regions of contact would be considered to be optimal in the sense discussed here. To express this idea formally, we now state the following.

Definition 3: Say we are given a set of pairs of independent regions of contact on edges $e_{1}$ and $e_{2}$ of a polygonal object, the $i$ th pair potentially defining a grasp named $g_{i}$. Denote this set by

$$
\begin{aligned}
\mathcal{S}=\left\{g_{1}:\left(s_{11}, s_{12}\right), g_{2}:\left(s_{21}, s_{22}\right), \cdots,\right. & \\
& \left.g_{i}:\left(s_{i 1}, s_{i 2}\right), \cdots\right\} .
\end{aligned}
$$

The optimal grasp, denoted $\left\{g^{\star}:\left(s_{1}^{\star}, s_{2}^{\star}\right)\right\}$ must satisfy

$$
\operatorname{MIN}\left\{d\left(s_{1}^{\star}\right), d\left(s_{2}^{\star}\right)\right\} \geq \operatorname{MIN}\left\{d\left(s_{i 1}\right), d\left(s_{i 2}\right)\right\}
$$

for all members of the set $\mathcal{S}$, where $d\left(s_{i}\right)$ is the length of the segment $s_{i}$. Of all the pairs $\left(s_{i 1}, s_{i 2}\right)$ that satisfy the above condition, the optimal grasp satisfies the following additional condition

$$
\operatorname{MAX}\left\{d\left(s_{1}^{\star}\right), d\left(s_{2}^{\star}\right)\right\} \geq \operatorname{MAX}\left\{d\left(s_{i 1}\right), d\left(s_{i 2}\right)\right\} .
$$

This definition, although cumbersome, espouses a very simple idea. To illustrate the underlying idea, assume that a grasp planner has returned a set of six possible two-fingered grasps for two designated surfaces of a polygonal object. We will denote each grasp by $g$ : $\left(d\left(s_{1}\right), d\left(s_{2}\right)\right)$, where $g$ is a grasp label and where $d\left(s_{1}\right)$ and $d\left(s_{2}\right)$ are the widths of the independent regions associated with the first and the second contacts, respectively. Assume for the sake of explanation

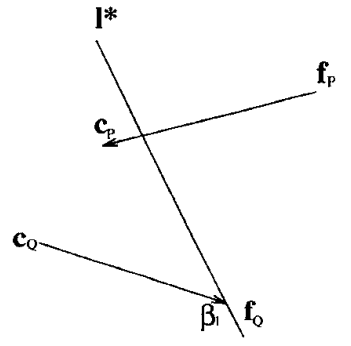

(a)

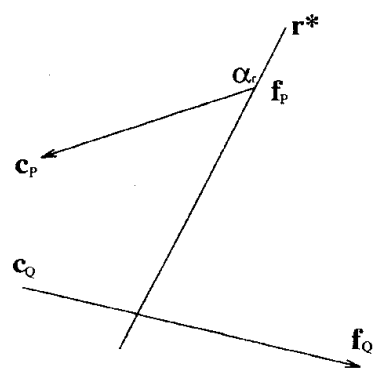

(b)

Fig. 11. For an optimal grasp, either $\mathbf{l}^{\star}$ passes through $\mathbf{f}_{Q}$ or $\mathbf{r}^{\star}$ passes through $\mathbf{f}_{P}$. (a) $\mathbf{l}^{\star}$ passes through $\mathbf{f}_{Q}$. (b) $\mathbf{r}^{\star}$ passes through $\mathbf{f}_{P}$.

that these six grasps are

$$
\begin{gathered}
\left\{g_{1}:(3,4), g_{2}:(2,1), g_{3}:(3,8),\right. \\
\left.g_{4}:(2,9), g_{5}:(1,5), g_{6}:(7,3) .\right\}
\end{gathered}
$$

The optimality criterion will first extract from each grasp the smaller of the segment widths and discard all those grasps whose values are less than the maximum of these widths. On this basis, the criterion will retain only the grasps: $\left\{g_{1}, g_{3}, g_{6}\right\}$. The optimality criterion will then extract from each of these grasps the larger of the segment values and discard all whose such values are less than the maximum. As a result, the grasp $g_{3}$ will be declared to be the optimal for this case. At this juncture, we would like to point the readers to some other possible criteria for optimum grasp synthesis under forceclosure. For example, Kirkpatrick et al. [17] and Ferrari and Canny [14] considered grasp configurations that are optimal with respect to applied finger forces.

\section{Generating Optimal Force-Closure Grasps}

This section presents algorithms for generating an optimal forceclosure grasp for a given pair of edges that satisfy the necessary criteria for force-closure (Section III) and that are in standard configuration, i.e., they have been assigned the distinguished names, $e_{P}$ and $e_{Q}$, and the associated parameterizations in accordance with the discussion in Section II. First, we state a property that is common to all optimal grasps.

Theorem 1: If the two independent regions of contact $\left(s_{P}^{\star}, s_{Q}^{\star}\right)$ define an optimal grasp on $e_{P}$ and $e_{Q}$, then there exists a grasp generating cone $C^{\star}\left(\mathrm{v}^{\star}\right)=\left(\mathrm{v}^{\star}, \mathrm{l}^{\star}, \mathbf{r}^{\star}\right)$ for $\left(s_{P}^{\star}, s_{Q}^{\star}\right)$ such that either $\alpha_{r}=0$ or $\beta_{l}=d_{Q}$.

In other words, for each optimal grasp there must exist a placement for the grasp generating cone $C^{\star}\left(\mathrm{v}^{\star}\right)$ such that either $\mathbf{l}^{\star}$ must pass through $\mathbf{f}_{Q}$ or $\mathbf{r}^{\star}$ must pass through $\mathbf{f}_{P}$, as shown in Fig. 11. Relative to the grasp generating cone $C^{\times}\left(\mathrm{v}^{\star}\right)$, there are three different situations for the two edges for which the independent regions of contact are nonempty: both edges are inside $C^{\times}\left(\mathbf{v}^{\star}\right)$, one edge in 
its entirety and a portion of the other edge lie inside $C^{\times}\left(\mathrm{v}^{\star}\right)$, or a portion of each edge lies inside $C^{\times}\left(\mathbf{v}^{\star}\right)$. More formally, we have

Theorem 2-Grasp Construction Theorem: The two independent regions of contact, $\left(s_{P}^{\star}, s_{Q}^{\star}\right)$, define an optimal grasp ${ }^{2}$ on $e_{P}$ and $e_{Q}$ if and only if there exists a grasp generating cone $C^{\times}\left(\mathbf{v}^{\star}\right)=$ $\left(\mathbf{v}^{\star}, 1^{\star}, \mathbf{r}^{\star}\right)$ for $\left(s_{P}^{\star}, s_{Q}^{\star}\right)$ such that

1) if $s_{Q}^{\star} \equiv e_{Q}$ and $s_{P}^{\star} \equiv e_{P}$, then $\beta_{r}=0, \beta_{l}=d_{Q}, \alpha_{r} \leq 0$, and $\alpha_{l} \geq d_{P}$

2) otherwise, if $s_{P}^{\star} \equiv e_{P}$, then $\alpha_{r}=0, \alpha_{l}=d_{P}$, and $d\left(s_{Q}^{\star}\right) \geq$ $d\left(s_{P}^{*}\right)$

3) otherwise, if $s_{Q}^{\star} \equiv e_{Q}$, then $\beta_{r}=0, \beta_{l}=d_{Q}$ and $d\left(s_{P}^{\star}\right) \geq$ $d\left(s_{Q}^{+}\right)$;

4) otherwise, $d\left(s_{P}^{\star}\right)=d\left(s_{Q}^{\star}\right)$ and either

$$
\begin{aligned}
& \text { a) } \alpha_{r}=0 \text { and } \beta_{l} \leq d_{Q} \quad \text { or } \\
& \text { b) } \beta_{l}=d_{Q} \text { and } \alpha_{r} \geq 0 .
\end{aligned}
$$

The proofs of both theorems are presented in the Appendix. Theorem 1 is subsumed by the grasp construction theorem, which is the basis of our algorithm for generating optimal force-closure grasps. The algorithm uses a hypothesize and verify approach. We start by supposing that $l^{\star}$ passes through $f_{Q}$. This situation is characterized by $\beta_{l}=d_{Q}$, which implies that one of conditional statements (1), (3), and (4b) of Theorem 2 is satisfied. The parameters associated with these conditional statements are then computed to verify the hypothesis. If none of these three conditions is satisfied, then the hypothesis about the location of $\mathrm{l}^{\star}$ is false. Instead, $\mathrm{r}^{\star}$ passes through $\mathbf{f}_{P}$, as shown in Fig. 11(b). This implies that $\alpha_{T}=0$, so either of the conditional statements (2) and (4a) is satisfied. The algorithm for generating the independent regions of contact that defines an optimal grasp is summarized below.

Algorithm 1-(Optimal Grasp Construction Algorithm):

The independent regions of contact that define an optimal grasp on two given edges can be constructed as follows

1) Apply the two necessary criteria for force closure presented in Section III. If either criterion is violated, then stop. There is no force-closure grasp for this pair of edges.

2) Convert the edges to their standard configuration using the algorithm presented in Section II-C and assign to them the distinguished labels $e_{P}$ and $e_{Q}$, together with the associated parameterizations.

3) By the method to be presented in Section V-A, find the unit vectors, $\mathbf{u}_{r}$ and $\mathbf{u}_{l}$, that characterize $\mathrm{r}^{\star}$ and $\mathbf{l}^{\star}$, the two bounding lines for the optimal placement of the GGC for the given $e_{P}$ and $e_{Q}$.

4) Using the procedure of Section V-B, check for the existence of a grasp generating cone for the edges $e_{P}$ and $e_{Q}$.

5) Assume that $\mathrm{l}^{\star}$ passes through $\mathrm{f}_{Q}$ and apply the algorithm which we will describe in Section V-C to verify this hypothesis. The verification process will generate the desired independent regions of contact if the hypothesis is valid.

6) If the above hypothesis is false, then $\mathbf{r}^{\star}$ passes through $\mathbf{f}_{P}$. The algorithm which we will present in Section V-D can be used to generate the independent regions of contact for the optimal grasp.

${ }^{2}$ In the case of parallel edges, generally there is no unique optimal grasp. In fact, there is potentially an infinite number of solutions that are optimal. The entire solution space is specified by the intersection of the projection of one edge onto the other.

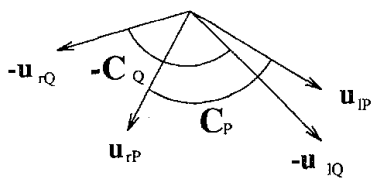

(a)
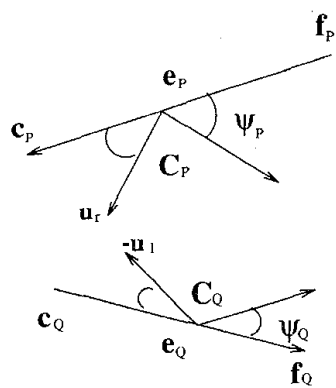

(b)

Fig. 12. The vectors $\mathbf{u}_{l}$ and $\mathbf{u}_{r}$ for the two edges $\mathbf{e}_{P}$ and $\mathbf{e}_{Q}$. (a) Intersecting $C_{P}$ and $-C_{Q}$. (b) $\mathbf{u}_{r}$ and $\mathbf{u}_{l}$ relative to $\mathbf{e}_{P}$ and $\mathbf{e}_{Q}$.

The first two steps of the algorithm have already been described in detail; the remaining steps of the algorithm will be described in order in the remainder of this section.

\section{A. Finding $\mathbf{u}_{r}$ and $\mathbf{u}_{l}$ Associated with $\mathbf{r}^{\star}$ and $\mathbf{1}^{\star}$}

The bounding lines $\mathrm{r}^{\star}$ and $\mathrm{l}^{\star}$ of the optimal placement of the GGC for the given $e_{P}$ and $e_{Q}$, must obviously come from the friction cones for the two edges. In keeping with the notation shown in Section II-A, the two unit vectors that characterize the boundaries of the friction cone $C_{P}$ for $e_{P}$ will be designated by $\mathbf{u}_{l P}$ and $\mathbf{u}_{r P}$. The two corresponding unit vectors for the friction cone $C_{Q}$ associated with $e_{Q}$ will be designated $\mathbf{u}_{l Q}$ and $\mathbf{u}_{r Q}$.

In accordance with our discussion in Section II-B, the values of $\mathbf{u}_{r}$ and $\mathrm{u}_{l}$ are obtained by computing the intersection of $C_{P}$ and $-C_{Q}$. Clearly, the value of $\mathbf{u}_{r}$ is given by $-\mathbf{u}_{r Q}$ if $-\mathbf{u}_{r Q}$ lies within $C_{P}$. Otherwise, it is given by $\mathbf{u}_{r P}$. Similarly, the value of $\mathbf{u}_{l}$ is given by either $-\mathbf{u}_{l Q}$ or $\mathbf{u}_{l P}$, depending on whether $-\mathbf{u}_{l Q}$ lies within $C_{P}$ or not. A pictorial illustration of this is given in Fig. 12.

Formally, the following two equations can be used to find $\mathbf{u}_{r}$ and $\mathrm{u}_{l}$ :

and

$$
\mathbf{u}_{l}= \begin{cases}-\mathbf{u}_{l Q} & \text { if } \mathbf{u}_{l Q} \times \mathbf{u}_{l P}<0 \\ \mathbf{u}_{l P} & \text { otherwise }\end{cases}
$$

$$
\mathbf{u}_{r}= \begin{cases}-\mathbf{u}_{r Q} & \text { if } \mathbf{u}_{r P} \times \mathbf{u}_{r Q}<0 \\ \mathbf{u}_{r P} & \text { otherwise. }\end{cases}
$$

For example, consider the edges $e_{P}$ and $e_{Q}$ shown in Fig. 12. The assignments $\mathbf{u}_{l} \equiv-\mathbf{u}_{l Q}$ and $\mathbf{u}_{r} \equiv \mathbf{u}_{r P}$ are made because $\mathbf{u}_{l Q} \times \mathbf{u}_{l P}<0$ and $\mathbf{u}_{r P} \times \mathbf{u}_{r Q}>0$, respectively.

\section{B. Checking for the Existence of a Grasp Generating Cone}

Once $\mathbf{u}_{l}$ and $\mathbf{u}_{r}$ are determined, it is possible to check if there exists a grasp generating cone that generates nonempty independent regions of contact. For two edges that satisfy the necessary criteria for force closure and that are in standard configuration, there are two situations in which the edges have no force-closure grasps, as shown in Fig. 13. The first case is shown in Fig. 13(a), where the right boundary $\mathbf{r}$ of the grasp generating cone passes through $\mathbf{f}_{Q}$, but the intersection of the grasp generating cone with the edge $e_{P}$ is empty. 


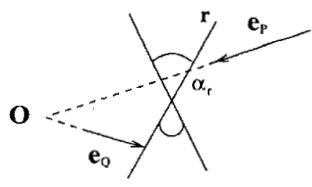

(a)

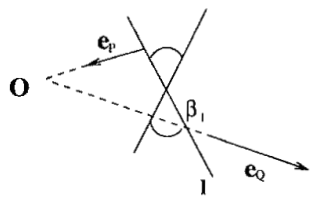

(b)

Fig. 13. Configurations with no force-closure grasps. (a) Case where $\alpha_{r} \geq d_{P}$. (b) Case where $\beta_{l} \leq 0$.

For this case, the intersection of the line $\mathbf{r}$ with the line containing $e_{P}$ is closer to $\mathbf{O}$ than $\mathbf{c}_{P}$ is, where $\mathbf{O}$ is the intersection of the lines containing $e_{P}$ and $\epsilon_{Q}$. Formally, this is represented by the predicate

$$
\text { NO-GRASP1: } \alpha_{r} \geq d_{P}
$$

where

$$
\begin{gathered}
\mathbf{f}_{P}+\alpha_{r} \mathbf{u}_{P}=\mathbf{f}_{Q}+\rho \mathbf{u}_{r} \\
\Rightarrow \alpha_{r}=\frac{\left(\mathbf{f}_{Q}-\mathbf{f}_{P}\right) \times \mathbf{u}_{r}}{\mathbf{u}_{P} \times \mathbf{u}_{r}} .
\end{gathered}
$$

The other case is depicted in Fig. 13(b). In this case, the left boundary $\mathbf{l}$ of the grasp generating cone passes through $\underline{f}_{P}$, but the intersection of the cone with the edge $e_{Q}$ is empty. For this situation, the intersection of the line 1 with the line containing $e_{Q}$ is closer to $\mathbf{O}$ than $\mathbf{c}_{Q}$ is, where $\mathbf{O}$ is the intersection of the lines containing $e_{P}$ and $e_{Q}$. Formally, this is captured by the predicate

$$
\text { NO-GRASP2: } \beta_{l} \leq 0
$$

where

$$
\begin{aligned}
& \mathbf{f}_{P}+\lambda \mathbf{u}_{l}=\mathbf{c}_{Q}+\beta_{l} \mathbf{u}_{Q} \\
& \Rightarrow \beta_{l}=\frac{\left(\mathbf{f}_{P}-\mathbf{c}_{Q}\right) \times \mathbf{u}_{l}}{\mathbf{u}_{Q} \times \mathbf{u}_{l}} .
\end{aligned}
$$

If either NO-GRASP1 or NO-GRASP2 is true, then there is no forceclosure grasp for the two edges. Otherwise, we use a hypothesize and verify method to generate an optimal grasp for the given edge-pair.

\section{C. $\mathbf{1}^{\star}$ Passes Through $\mathbf{f}_{Q}$}

We start by assuming $I^{\star}$ passes through $f_{Q}$. This implies that one of the conditional statements labeled (1), (3), and (4b) in the grasp construction theorem (Theorem 2) is satisfied. Then, we verify the validity of this supposition. If it is valid, then the verification process generates the optimal grasp. Otherwise, the algorithm to be described in Section V-D is applied to generate the optimal grasp.

Applying Conditions (1) and (3) of the Theorem: We first consider the case for which either condition (1) or (3) is satisfied, i.e., edge $e_{Q}$ is entirely within $C^{\times}\left(\mathbf{v}^{\star}\right)$ as shown in Fig. 14. To check if either of these two conditions is satisfied, we need to compute the parameters $\alpha_{l}$ and $\alpha_{r}$.

The values of $\alpha_{l}$ and $\alpha_{r}$ are obtained by computing the intersection point for $\mathbf{l}^{\star}$ with $e_{T}$, and the intersection point of $\mathbf{r}^{\star}$ with $e_{P}$,

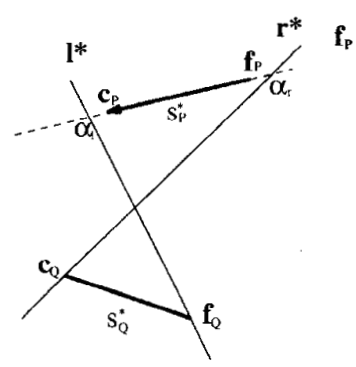

(a)

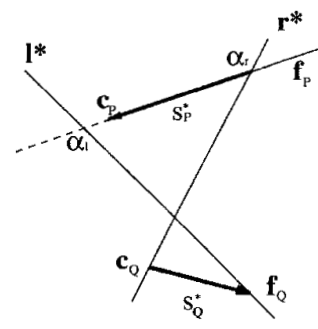

(b)

Fig. 14. $s_{Q}^{\star}$ is inside $C^{\times}\left(\mathbf{v}^{\star}\right)$. (a) Condition (1) is satisfied. (b) Condition (3) is satisfied.

respectively:

$$
\begin{aligned}
\mathbf{f}_{Q}+\lambda \mathbf{u}_{l} & =\mathbf{f}_{P}+\alpha_{l} \mathbf{u}_{P} \\
\Rightarrow \alpha_{l} & =\frac{\left(\mathbf{f}_{Q}-\mathbf{f}_{P}\right) \times \mathbf{u}_{l}}{\mathbf{u}_{P} \times \mathbf{u}_{l}} \\
\mathbf{c}_{Q}+\rho \mathbf{u}_{r} & =\mathbf{f}_{P}+\alpha_{r} \mathbf{u}_{P} \\
\Rightarrow \alpha_{r} & =\frac{\left(\mathbf{c}_{Q}-\mathbf{f}_{P}\right) \times \mathbf{u}_{r}}{\mathbf{u}_{P} \times \mathbf{u}_{r}}
\end{aligned}
$$

If the following predicate is satisfied, then condition (1) of Theorem 2 applies:

$$
\text { COND-1-SATISFIED: } \alpha_{r} \leq 0 \& \alpha_{l} \geq d_{P} \text {. }
$$

In this case, the optimal grasp is defined by $s_{P}^{\star} \equiv e_{P}$ and $s_{Q}^{\star} \equiv e_{Q}$. Otherwise, condition (1) is not satisfied, so we proceed to check for whether condition (3) is met.

Condition (3) is satisfied if the following predicate is true:

COND-3-SATISFIED: $\operatorname{MIN}\left\{d_{P}, \alpha_{l}\right\}-\operatorname{MAX}\left\{0, \alpha_{r}\right\} \geq d_{Q}$.

The MAX and MIN operators are needed to handle the cases in which $\mathbf{c}_{P}$ or $\mathbf{f}_{P}$ lie inside of the grasp generating cone. If COND-3-SATISFIED is true, the end points of $s_{P}^{\star}$ are specified by $\left\{\operatorname{MIN}\left\{d_{P}, \alpha_{l}\right\}, \operatorname{MAX}\left\{0, \alpha_{r}\right\}\right\}$. Otherwise, condition (3) is not satisfied. In this case, we check to see if condition (4b) is satisfied.

Applying Condition (4b) of Theorem 2: Suppose condition (4b) applies, then neither edge will be entirely within the optimal placement of the grasp generating cone. As shown in Fig. 15, two different situations correspond to this case $-\mathbf{c}_{P}$ is either outside of $C^{\times}\left(\mathrm{v}^{\star}\right)$, as shown in Fig. 15(a), or it is within $C^{\times}\left(\mathrm{v}^{\star}\right)$, as shown in Fig. 15(b).

In both of the situations depicted in Fig. 15(a) and (b), we have $d\left(s_{P}^{\star}\right)=d\left(s_{Q}^{\star}\right)$. For the former situation, we express the intersection points of $\mathbf{r}^{\star}$ with $e_{Q}$ and $e_{P}$ as $\mathbf{f}_{Q}+\beta_{r 1} \mathbf{u}_{Q}$ and $\mathbf{f}_{P}+\left(\alpha_{l}+\beta_{r l}\right) \mathbf{u} P$, respectively. Thus, we have

$$
\begin{aligned}
\mathbf{f}_{Q}+\beta_{r 1} \mathbf{u}_{Q}+\rho \mathbf{u}_{r} & =\mathbf{f}_{P}+\left(\alpha_{l}+\beta_{r 1}\right) \mathbf{u}_{P} \\
\Rightarrow \beta_{r 1}\left(\mathbf{u}_{P}-\mathbf{u}_{Q}\right) & =\mathbf{f}_{Q}-\mathbf{f}_{P}-\alpha_{l} \mathbf{u}_{P}+\rho \mathbf{u}_{r} \\
\Rightarrow \beta_{r 1} & =\frac{\left(\mathbf{f}_{Q}-\mathbf{f}_{P}-\alpha_{l} \mathbf{u}_{P}\right) \times \mathbf{u}_{r}}{\left(\mathbf{u}_{P}-\mathbf{u}_{Q}\right) \times \mathbf{u}_{r^{r}}} .
\end{aligned}
$$

The value of $\alpha_{l}$ is given by (1). 


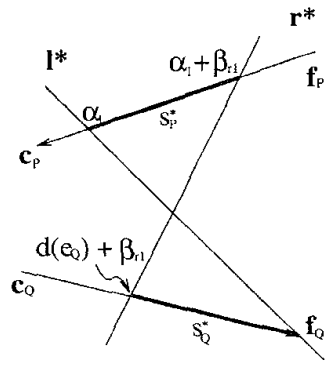

(a)

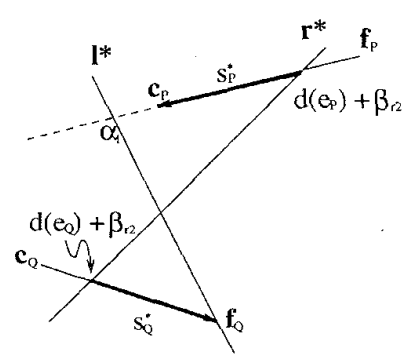

(b)

Fig. 15. Situations in which condition (4b) of the theorem applies. (a) $c_{P}$ is outside of $C^{\times}\left(\mathbf{v}^{\star}\right)$. (b) $c_{P}$ is within $C^{\times}\left(\mathrm{v}^{\star}\right)$.

At this point, we can verify the hypothesis that condition (4b) in Theorem 2 is satisfied. The predicate

$$
\text { COND-4B-SATISFIED: } \alpha_{l}+\beta_{r 1} \geq 0
$$

evaluates to true if condition (4b) of the theorem is satisfied. To check which of the two different situations discussed above is true we compute the predicate

$$
\text { COND-4B-1-SATISFIED: } \alpha_{r} \leq d_{P} \text {. }
$$

If COND-4B-1-SATISFIED is true, then the situation is as depicted in Fig. 15(a). In this case, $s_{P}^{\star}$ and $s_{Q}^{\star}$ are characterized by the parameters $\left\{\alpha_{l}+\beta_{r 1}, \alpha_{l}\right\}$ and $\left\{d_{Q}+\beta_{r 1}, d_{Q}\right\}$, respectively.

Otherwise, the situation is as depicted in Fig. 15(b). For this case, we can express the intersection of $\mathrm{r}^{\star}$ with $e_{Q}$ and $e_{P}$ as $\left\{\mathbf{f}_{Q}+\beta_{r^{\prime}} \mathbf{u}_{Q}\right\}$ and $\left\{\mathbf{c}_{P}+\beta_{r 2} \mathbf{u}_{P}\right\}$, respectively. Thus, we have

$$
\begin{aligned}
\mathbf{f}_{Q}+\beta_{r 2} \mathbf{u}_{Q}+\rho \mathbf{u}_{r} & =\mathbf{c}_{P}+\beta_{r 2} \mathbf{u}_{P} \\
\Rightarrow \beta_{r 2} & =\frac{\left(\mathbf{f}_{Q}-\mathbf{c}_{P}\right) \times \mathbf{u}_{r}}{\left(\mathbf{u}_{P}-\mathbf{u}_{Q}\right) \times \mathbf{u}_{r}} .
\end{aligned}
$$

In this case, $s_{P}^{\star}$ and $s_{Q}^{\star}$ are characterized by $\left\{d_{P}+\beta_{r 2}, d_{P}\right\}$ and $\left\{d_{Q}+\beta_{r 2}, d_{Q}\right\}$, respectively.

This completes our derivation of the optimal grasp for the case where $I^{\star}$ passes through $f_{Q}$. The following procedure summarizes our discussion in this section.

Algorithm 2-( $1^{\star}$ Algorithm $)$ : The algorithm for handling the case in which $\mathbf{l}^{\star}$ passes through $\mathbf{f}_{Q}$ is as follows:

1) Compute $\alpha_{l}$ and $\alpha_{r}$ using (1) and (2), respectively. They define the predicates COND-1-SATISFIED and COND-3-SATISFIED.

2) If COND-1-SATISFIED is true, then the conditional statement (1) of Theorem 2 applies. The optimal grasp is defined by $s_{P}^{\star} \equiv e_{P}$ and $s_{Q}^{\star} \equiv e_{Q}$.

3) Otherwise, if COND-3-SATISFIED is true, then the conditional statement (3) of Theorem 2 applies. The optimal grasp is

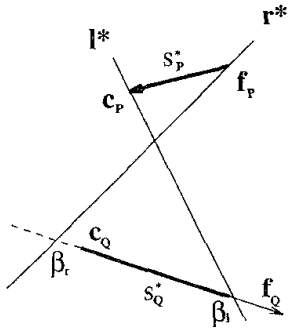

Fig. 16. A situation in which condition (2) applies.

defined by

$$
\begin{cases}s_{P}^{\star}: & \left\{\operatorname{MAX}\left\{0, \alpha_{r}\right\}, \operatorname{MIN}\left\{\alpha_{l}, d_{P}\right\},\right\} \\ s_{Q}^{\star}: & \left\{0, d_{Q}\right\} .\end{cases}
$$

4) Otherwise, compute the value of the predicate COND-4BSATISFIED. If it is false, then the hypothesis that $l^{\star}$ passes through $\mathbf{f}_{Q}$ is invalid. In this case, the optimal grasp is determined using the algorithm described in the next section. Otherwise, if the predicate COND-4B-1-SATISFIED evaluates to true, then the optimal grasp is defined by $\alpha_{l}$ and $\beta_{r 1}$, i.e.,

$$
\begin{cases}s_{P}^{\star}: & \left\{\alpha_{l}+\beta_{r 1}, \alpha_{l}\right\} \\ s_{Q}^{\star}: & \left\{d_{Q}+\beta_{r 1}, d_{Q}\right\}\end{cases}
$$

where $\beta_{r 1}$ is given by (3). Otherwise, the optimal grasp is defined by

$$
\begin{cases}s_{P}^{\star}: & \left\{d_{P}+\beta_{r 2}, d_{P}\right\} \\ s_{Q}^{\star}: & \left\{d_{Q}+\beta_{r 2}, d_{Q}\right\}\end{cases}
$$

where $\beta_{r^{2}}$ is given by (4).

\section{Finding Optimal Grasp Given $\mathbf{r}^{\star}$}

In this section, we will describe the method for determining an optimal grasp for the case in which $\mathbf{r}^{\star}$ is known, i.e., $\mathbf{r}^{\star}$ passes through $\mathrm{f}_{P}$, or

$$
\mathbf{r}^{\star}=\mathbf{f}_{P}+\rho \mathbf{u}_{r} .
$$

The algorithm described in this section is invoked when the predicates COND-1-SATISFIED, COND-3-SATISFIED, and COND-4BSATISFIED all evaluate to false. In this case, either of the conditional statements (2) and (4a) in Theorem 2 applies. Again, a hypothesize and verify strategy is used for grasp synthesis. We first assume that the conditional statement (2) applies and then verify that this is true. If this hypothesis is false, then the conditional statement (4a) of the theorem is satisfied. All that remains is to invoke the appropriate method to generate the grasp.

Applying Condition (2) of the Theorem: First of all, let us suppose that condition (2) is satisified. Fig. 16 shows a situation that satisfies this condition. To verify our supposition, we compute the values of $\beta_{r}$ and $\beta_{l}$.

The value of $\beta_{r}$ characterizes the intersection of $\mathbf{r}^{\star}$ and $e_{Q}$, and may be computed by the following:

$$
\begin{gathered}
\mathbf{f}_{P}+\rho \mathbf{u}_{r}=\mathbf{c}_{Q}+\beta_{r} \mathbf{u}_{Q} \\
\Rightarrow \beta_{r}=\frac{\left(\mathbf{f}_{P}-\mathbf{c}_{Q}\right) \times \mathbf{u}_{r}}{\mathbf{u}_{Q} \times \mathbf{u}_{r}} .
\end{gathered}
$$

The value of $\beta_{l}$ can be computed in a similar manner:

$$
\begin{gathered}
\mathbf{c}_{P}+\lambda \mathbf{u}_{l}=\mathbf{c}_{Q}+\beta_{l} \mathbf{u}_{Q} \\
\Rightarrow \beta_{l}=\frac{\left(\mathbf{c}_{P}-\mathbf{c}_{Q}\right) \times \mathbf{u}_{l}}{\mathbf{u}_{Q} \times \mathbf{u}_{l}} .
\end{gathered}
$$




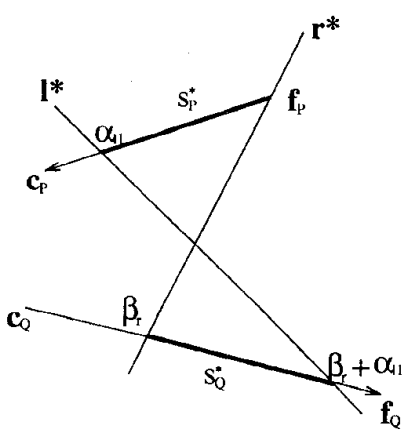

(a)

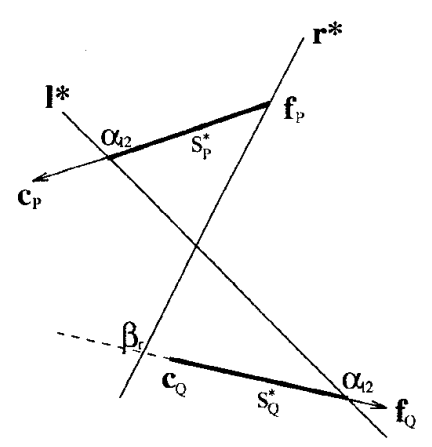

(b)

Fig. 17. The situations in which condition (4a) of the theorem is satisfied. (a) $\mathbf{c}_{Q}$ is outside of $C^{\times}\left(\mathbf{v}^{\star}\right)$. (b) $\mathbf{c}_{Q}$ is within $C^{\times}\left(\mathbf{v}^{\star}\right)$.

The hypothesis is valid if the following predicate is true:

$$
\text { COND-2-SATISFIED: } \operatorname{MIN}\left\{d_{Q}, \beta_{l}\right\}-\operatorname{MAX}\left\{0, \beta_{r}\right\} \geq d_{P} \text {. }
$$

In this case, the end points of $s_{Q}^{\star}$ are specified by $\left.\left\{\operatorname{MAX}\left\{\beta_{T}, 0\right)\right\}, \operatorname{MIN}\left\{\beta_{l}, d_{Q}\right\}\right\}$. Otherwise, condition (4a) of Theorem 2 is satisfied.

Applying Condition (4a) of the Theorem: Since condition (4a) of the theorem is satisifed, neither edge will be entirely within the optimal grasp generating cone $C^{\times}\left(\mathbf{v}^{*}\right)$. There are two different situations for this case, as shown in Fig. 17, with $\mathrm{c}_{Q}$ within $C^{\star}\left(\mathrm{v}^{\star}\right)$ for Fig. 17(a) and $c_{Q}$ outside of $C^{\times}\left(v^{\star}\right)$ for Fig. 17(b). The former situation is characterized by the predicate

$$
\text { COND-4A-1-SATISFIED: } \beta_{r} \geq 0
$$

where $\beta_{r}$ is given by (5). If COND-4A-1-SATISFIED evaluates to false, then the latter situation is true.

Suppose COND-4A-1-SATISFIED evaluates to true, i.e., the situation is as depicted in Fig. 17(a). Since $\mathbf{r}^{\star}$ passes through $\mathbf{f}_{P}$ and $d\left(s_{P}^{\star}\right)=d\left(s_{Q}^{\star}\right)$, we can express the intersection of $l^{\star}$. with $e_{P}$ and $e_{Q}$, respectively, as $\left\{\mathbf{f}_{P}+\alpha_{l 1} \mathbf{u}_{P}\right\}$ and $\left\{\mathbf{c}_{Q}+\left(\beta_{r}+\alpha_{l 1}\right) \mathbf{u}_{Q}\right\}$. Thus, we have

$$
\begin{aligned}
\mathbf{f}_{P}+\alpha_{l 1} \mathbf{u}_{P}+\lambda \mathbf{u}_{l} & =\mathbf{c}_{Q}+\left(\beta_{T}+\alpha_{l 1}\right) \mathbf{u}_{Q} \\
\Rightarrow \alpha_{l 1}\left(\mathbf{u}_{P}-\mathbf{u}_{Q}\right) & =\mathbf{c}_{Q}-\mathbf{f}_{P}+\beta_{r} \mathbf{u}_{Q}-\lambda \mathbf{u}_{l} \\
\Rightarrow \alpha_{l 1} & =\frac{\left(\mathbf{c}_{Q}-\mathbf{f}_{P}+\beta_{r} \mathbf{u}_{Q}\right) \times \mathbf{u}_{l}}{\left(\mathbf{u}_{P}-\mathbf{u}_{Q}\right) \times \mathbf{u}_{l}} .
\end{aligned}
$$

If COND-4A-1-SATISFIED is false, then the situation is as shown by Fig. 17(b). For this case, we express the intersection of $1^{\star}$ with $e_{P}$ and $e_{Q}$ as $\mathbf{f}_{P}+\alpha_{l 2} \mathbf{u}_{P}$ and $\mathbf{c}_{Q}+\alpha_{l_{2}} \mathbf{u}_{Q}$, respectively. Thus, we have

$$
\begin{aligned}
\mathbf{f}_{P}+\alpha_{l 2} \mathbf{u}_{P}+\lambda \mathbf{u}_{l} & =\mathbf{c}_{Q}+\alpha_{l 2} \mathbf{u}_{Q} \\
\Rightarrow \alpha_{l 2} & =\frac{\left(\mathbf{c}_{Q}-\mathbf{f}_{P}\right) \times \mathbf{u}_{l}}{\left(\mathbf{u}_{P}-\mathbf{u}_{Q}\right) \times \mathbf{u}_{l}} .
\end{aligned}
$$

Algorithm 3-( $\mathbf{r}^{\star}$ Algorithm $)$ : The algorithm for handling the cases in which $\mathbf{r}^{\star}$ passes through $\mathbf{f}_{P}$ is as follows.

1) Compute $\beta_{r}$ and $\beta_{l}$ using Equations (5) and (6), respectively. They define the predicate COND-2-SATISFIED.

2) If COND-2-SATISFIED is true, then the optimal grasp is defined by

$$
\begin{cases}s_{P}^{\star}: & \left\{0, d_{P}\right\} \\ s_{Q}^{\star}: & \left\{\operatorname{MAX}\left\{\beta_{r}, 0\right\}, \operatorname{MIN}\left\{d_{Q}, \beta_{l}\right\}\right\} .\end{cases}
$$

3) Otherwise, condition (4a) of the theorem is satisfied. If the predicate COND-4A-1-SATISFIED is true, then the optimal grasp is defined by

$$
\begin{cases}s_{P}^{\star}: & \left\{0, \alpha_{l 1}\right\} \\ s_{Q}^{\star}: & \left\{\beta_{r}, \beta_{r}+\alpha_{l 1}\right\}\end{cases}
$$

where $\alpha_{l 1}$ is given by (7). Otherwise, the optimal grasp is defined by

$$
\begin{cases}s_{P}^{\star}: & \left\{0, \alpha_{l 2}\right\} \\ s_{Q}^{\star}: & \left\{0, \alpha_{l 2}\right\}\end{cases}
$$

where $\alpha_{l 2}$ is given by (8).

\section{Grasp Generation Algorithm}

An optimal grasp for a polygon is the grasp that tolerates the largest positioning errors for each fingertips at the contact points. To find this grasp, exhaustively pair all the edges of the polygon and apply the Grasp Construction Algorithm described above. The optimal grasp for the polygon is then defined by the independent regions of contact that has the largest minimal length. A straightforward approach can generate the optimal force-closure grasp for the object in $O\left(n^{2}\right)$ time, since finding the set of independent regions of contact for a polygonal object with $n$ edges requires the enumeration of $\frac{1}{2}\left(\begin{array}{l}n \\ 2\end{array}\right)$ combinations, and so costs $O\left(n^{2}\right)$ time.

\section{Force-Closure Grasps in 3-D}

In order to generate force-closure grasps with two contact points for three-dimensional polyhedral objects, we will assume the contacts to be soft-finger contacts. With this assumption, the properties of a force-closure grasp is similar to the case for 2-D polygonal objects.

Theorem 3: The grasp with two soft-finger contacts at $P$ and $Q$ is a force-closure grasp if and only if the segment $\overline{P Q}$ lies strictly within the friction cones at $P$ and $Q$ [15].

The necessary criteria for force closure, with slight modifications, also hold for the 3-D case. Instead of grasping two edges, the grasp now contacts two faces of the polyhedral. The necessary criteria for force closure for the 3-D case are as follows.

1) The angle between the outward normals of the two faces must satisfy

$$
|\theta|>\psi_{1}+\psi_{2}
$$

where $\theta=\cos ^{-1}\left(\mathbf{n}_{1} \cdot \mathbf{n}_{2}\right)$ and $\psi_{i}$ is the magnitude of the angle between face $i$ and the boundary of its friction cone.

2) If the material side of a face is considered its "front," then there must exist two points, one on each face, such that the segment connecting these two points is either in front of both faces or behind both faces.

If any pair of faces fails to satisfy any of these two conditions, then it would not have any force-closure grasp.

The problem of finding the optimal force-closure grasp for a pair of faces that satisfy the necessary criteria for force closure in 3$\mathrm{D}$ involves intersecting the grasp generating cone, which now is 
formed from the intersection of two three-dimensional cones, with two polygons in 3-D space such that the resulting areas of intersection are optimal. This is extremely difficult to compute for faces which are polygons of arbitrary convexity in three-dimensional space. However, an approximate solution can be obtained easily.

For a pair of faces that satisfy the necessary criteria for force closure in 3-D, the algorithm for the 2-D case can be used to find force-closure grasps for these two faces. To simplify the calculation, we represent each nonconvex face with a set of maximal convexes that spans the face. The algorithm for finding a force-closure grasp for two faces $p_{1}$ and $p_{2}$ is simple and straightforward.

1) Rotate the face $\mathbf{p}_{2}$ to get a new face $\hat{\mathbf{p}}_{2}$, which is coplanar with the face $p_{1}$, i.e.

$$
\hat{\mathbf{p}}_{2}=\operatorname{rot}_{1}\left(\theta, \mathbf{p}_{2}\right)
$$

where $l$ is the line obtained by intersecting the planes containing $\mathbf{p}_{1}$ and $\mathbf{p}_{2}$.

2) Compute the intersections of $\hat{\mathrm{p}}_{2}$ and $\mathbf{p}_{1}$. Now, find the two points of the resultant polygon that have the longest distance between any pair of points of the polygon. Since both $\hat{\mathbf{p}}_{2}$ and $p_{1}$ are convex, their intersection is also convex. Therefore, the longest distance is always between two nonadjacent vertices. Denote these two points by $\mathbf{A}_{1}$ and $\mathbf{B}_{1}$.

3) Find the corresponding points of $\left(\mathbf{A}_{1}, \mathbf{B}_{1}\right)$ in face $\mathbf{p}_{2}$. Let $\left(\mathbf{A}_{2}, \mathbf{B}_{2}\right)$ be such points, i.e., $\mathbf{A}_{2}=\operatorname{rot}_{1}\left(-\theta, \mathbf{A}_{1}\right)$ and $\mathbf{B}_{2}=$ $\operatorname{rot}_{1}\left(-\theta, \mathbf{B}_{1}\right)$. These four points will give two segments, $\overline{\mathbf{A}_{1} \mathbf{B}_{1}}$ and $\overline{\mathbf{A}_{2} \mathbf{B}_{2}}$, that lie in the interior of the faces $p_{1}$ and $\mathbf{p}_{2}$, respectively.

4) Use the algorithm for the 2-D case on these two segments. The grasp defined on these two segments is also a force-closure grasp on the two faces. Recall that the equations for computing the parameters of the independent regions of contact turned out to be ratios of two cross-products, i.e., they had the form

$$
\frac{a \times b}{c \times d} \text {. }
$$

In the 3-D case, the $x$ operator is the usual cross-product in 3-D Euclidean space. If we were to rederive the formulas for the various parameters, the final results would be ratios of two dot product terms:

$$
\frac{(\mathbf{a} \times \mathbf{b}) \cdot(\mathbf{c} \times \mathbf{d})}{(\mathbf{c} \times \mathbf{d}) \cdot(\mathbf{c} \times \mathbf{d})}
$$

\section{CONCLUSION}

We have formulated and proved a new theorem that resulted in an efficient algorithm for computing two-fingered force-closure grasps for arbitrary polygonal objects. Besides being simple, efficient and elegant, it is the only algorithm that can generate force-closure grasps consisting of independent regions of contact for arbitrary polygons of arbitrary material property.

\section{APPENDIX}

We first present a simple lemma.

Lemma 1-Translational Lemma: For two semiinfinite edges $\vec{e}_{P}$ and $\vec{e}_{Q}$ that are in standard configuration and that satisfy the necessary criteria for force closure, the independent regions of contact generated by any two of grasp generating cones $C^{\times}(a \mathbf{u})=\{a \mathbf{u}, \mathbf{l}, \mathbf{r}\}$ and $C^{\times}((a+\delta) \mathbf{u})=\left\{(a+\delta) \mathbf{u}, \mathbf{l}^{\prime}, \mathbf{r}^{\prime}\right\}$ satisfy the following relationships:

1) if $\delta>0$, then $d\left(s_{P}\right)<d\left(s_{P}^{+}\right)$and $d\left(s_{Q}\right)<d\left(s_{Q}^{+}\right)$;

2) if $\delta<0$, then $d\left(s_{P}\right)>d\left(s_{P}^{+}\right)$and $d\left(s_{Q}\right)>d\left(s_{Q}^{+}\right)$

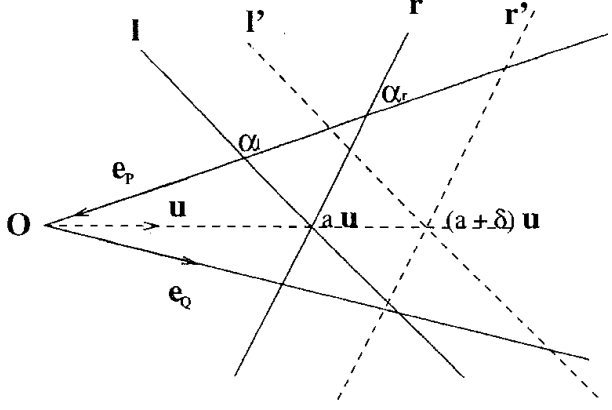

Fig. 18. Translating $C^{\times}(a \mathbf{u})$ to $C^{\times}((a+\delta) \mathbf{u})$.

where $\mathbf{u}$ is some convex sum of $-\mathbf{u}_{P}$ and $\mathbf{u}_{Q} ; s_{x}$ and $s_{x}^{+}$are the portions of edge $x \in\{P, Q\}$, that lies inside the grasp generating cones $C^{\times}(a \mathbf{u})$ and $C^{\times}((a+\delta) \mathbf{u})$, respectively. The constant $\delta$ is known as the translation constant.

Proof: Without loss of generality, let's assume that the intersection point $\mathbf{O}$ of $\vec{e}_{P}$ and $\vec{e}_{Q}$ is at the origin, as illustrated in Fig. 18. Thus, $\vec{e}_{P}$ and $\vec{e}_{Q}$ can be represented by the sets

$$
\left\{\alpha \mathbf{u}_{P} \mid \alpha \leq 0\right\} \text { and }\left\{\beta \mathbf{u}_{Q} \mid \beta \geq 0\right\}
$$

respectively. We can also represent the boundaries of the grasp generating cone $C^{\times}(a \mathrm{u})$ by the following two equations:

$$
\begin{aligned}
& \mathbf{l}=a \mathbf{u}+\lambda \mathbf{u}_{l} \\
& \mathbf{r}=a \mathbf{u}+\rho \mathbf{u}_{r}
\end{aligned}
$$

where $\mathbf{u}_{l}$ and $\mathbf{u}_{r}$ are some unit vectors lying on $\mathbf{l}$ and $\mathbf{r}$, respectively. Now, we can express the length of $s_{P}$ as $f(a)$, a function of $a$. The intersection between $\mathbf{I}$ and $\vec{e}_{P}$ is expressed by the relationship

$$
a \mathbf{u}+\lambda_{P} \mathbf{u}_{l}=\alpha_{l} \mathbf{u}_{P}
$$

$$
\Rightarrow \alpha_{l}=\frac{a \mathbf{u} \times \mathbf{u}_{l}}{\mathbf{u}_{P} \times \mathbf{u}_{l}} .
$$

Similarly, we obtain the intersection between $\mathbf{r}$ and $\vec{e}_{P}$ :

$$
\begin{gathered}
\alpha_{r}=\frac{a \mathbf{u} \times \mathbf{u}_{r}}{\mathbf{u} P \times \mathbf{u}_{r}} . \\
\Rightarrow f(a)=\alpha_{l}-\alpha_{r}=a\left(\frac{\mathbf{u} \times \mathbf{u}_{l}}{\mathbf{u}_{P} \times \mathbf{u}_{l}}-\frac{\mathbf{u} \times \mathbf{u}_{r}}{\mathbf{u}_{P} \times \mathbf{u}_{r}}\right) .
\end{gathered}
$$

Therefore, the lengths of the segments $s p$ and $s_{P}^{+}$are

$$
d(s p)=f(a)
$$

and

$$
d\left(s_{P}^{+}\right)=f(a+\delta)=f(a)+f(\delta)
$$

respectively. Since $f(\delta)>0$ if $\delta>0$ and $f(\delta)<0$ if $\delta<0$, we have

$$
\left\{\begin{array}{l}
d\left(s_{P}^{+}\right)>d\left(s_{P}\right) \text { if } \delta>0 \\
d\left(s_{P}^{+}\right)<d\left(s_{P}\right) \text { if } \delta<0 .
\end{array}\right.
$$

We can use similar arguments to establish the displacement relationship between $s_{Q}^{+}$and $s_{Q}$.

Q.E.D.

Lemma 1 also holds for the case for two edges of finite length. The only difference is that for the cases where $\alpha_{r}<0$ or $\beta_{l}>d_{p}$, a positive translation will cause a decrease in length, while a negative translation (until either $\alpha_{r}=0$ or $\beta_{l}=d_{P}$ ) will cause an increase in length. 


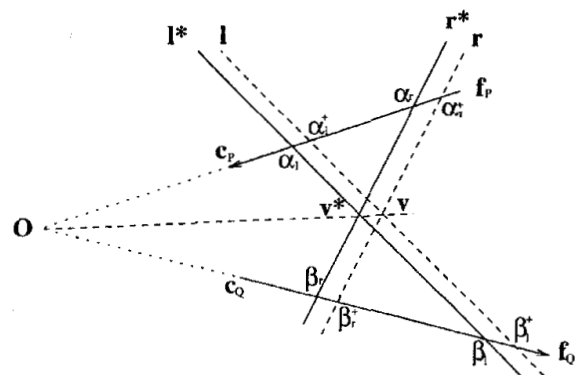

Fig. 19. A situation in which $C^{\times}\left(\mathbf{v}^{\star}\right)$ intersects neither $\mathbf{f}_{P}$ nor $\mathbf{f}_{Q}$.

Proof for Theorem 1: Theorem: If the two independent regions of contact $\left(s_{P}^{\star}, s_{Q}^{\star}\right)$ define an optimal grasp on $e_{P}$ and $\epsilon_{Q}$, then there must exist a grasp generating cone $C^{\times}=\left\{\mathbf{v}^{\star}, l^{\star}, \mathbf{r}^{\star}\right\}$ for $\left(s_{P}^{\star}, s_{Q}^{\star}\right)$ such that either $\alpha_{r}=0$ or $\beta_{l}=d_{Q}$.

Proof: Theorem 1 is really a corollary of the Translation Lemma. Suppose that $\left(s_{P}^{\star}, s_{Q}^{\star}\right)$ defines an optimal grasp, but it has no grasp generating cone such that either $\alpha_{r}=0$ or $\beta_{l}=d_{Q}$.

Let $C^{\times}\left(\mathbf{v}^{\star}\right)=\left\{\mathbf{v}^{\star}, \mathbf{l}^{\star}, \mathbf{r}^{\star}\right\}$ be a grasp generating cone for $\left(s_{P}^{\star}, s_{Q}^{\star}\right)$. Then there are two possibilities: $\alpha_{r}<0$ or $\alpha_{r}>0$. Suppose the former case is true. Then the independent regions of contact $\left(s_{P}, s_{Q}\right)$ generated by the grasp generating cone

$$
C^{\times}(\mathbf{v})=\left\{\mathbf{v}, \mathbf{l}^{\star}, \mathbf{r}=\mathbf{f}_{P}+\beta \mu_{r}\right\}
$$

satisfy

$$
\begin{gathered}
d\left(s_{P}\right)=d\left(s_{P}^{\star}\right) \\
d\left(s_{Q}\right) \geq d\left(s_{Q}^{\star}\right) .
\end{gathered}
$$

If the other situation is true, i.e., $\alpha_{r}>0$, then there are two cases to consider: $\beta_{l}<d_{P}$ or $\beta_{l}>d_{P}$. We can use similar arguments to those given above to show that the latter case cannot correspond to an optimal grasp. So we only need to consider the case in which $\alpha_{r}>0$ and $\beta_{l}<d_{P}$. For this situation, we can construct another grasp generating cone $C^{\times}(\mathbf{v})=\{\mathbf{v}, \mathbf{l}, \mathbf{r}\}$ by translating the vertex of $C^{\times}\left(\mathbf{v}^{\star}\right)$ by a small increment $\delta$ along the line $\overline{\mathrm{Ov}}$ away from $\mathrm{O}$ such that 1 and $\mathbf{r}$ intersects $e_{P}$ and $e_{Q}$, respectively:

$$
\left\{\begin{array}{l}
\mathrm{v}=\mathrm{v}^{\star}+\delta\left|\mathbf{v}^{\star}-\mathbf{O}\right|, \alpha_{r}>\alpha_{r}^{+}>0 \\
\beta_{l}<\beta_{l}^{+}<d_{P}
\end{array}\right.
$$

where $\alpha_{r}^{+}$and $\beta_{l}^{+}$parameterize the intersections of $\mathbf{r}$ and $e_{P}$ and 1 and $e_{Q}$, respectively. A pictorial illustration of this is given in Fig. 19. By the Translation Lemma:

$$
\begin{aligned}
d\left(s_{P}\right) & >d\left(s_{P}^{\star}\right) \\
d\left(s_{Q}\right) & >d\left(s_{Q}^{\star}\right) . \\
& \Rightarrow \Leftarrow
\end{aligned}
$$

Proof for Theorem 2: We will now re-state the Grasp Construction Theorem and present its proof.

Theorem: For any two edges that are in standard configuration and that satisfy the necessary criteria for force closure, the two independent regions of contact, $\left(s_{P}^{\star}, s_{Q}^{\star}\right)$, define an optimal grasp on $e_{P}$ and $e_{Q}$ if and only if there exists a grasp generating cone $C^{\times}\left(\mathbf{v}^{\star}\right)=\left(\mathbf{v}^{\star}, \mathbf{l}^{\star}, \mathbf{r}^{\star}\right)$ for $\left(s_{P}^{\star}, s_{Q}^{\star}\right)$ such that

1) if $s_{Q}^{\star} \equiv e_{Q}$ and $s_{P}^{\star} \equiv e_{P}$, then $\beta_{l}=d_{Q}, \beta_{r}=0, \alpha_{r} \leq 0$, and $\alpha_{l} \geq d_{P}$

2) otherwise, if $s_{P}^{\star} \equiv e_{P}$, then $\alpha_{r}=0, \alpha_{l}=d_{P}$, and $d\left(s_{Q}^{\star}\right) \geq$ $d\left(s_{P}^{*}\right)$

3) otherwise, if $s_{Q}^{\star} \equiv e_{Q}$, then $\beta_{t}=d_{Q}, \beta_{r}=0$ and $d\left(s_{P}^{\star}\right) \geq$ $d\left(s_{Q}^{\star}\right)$

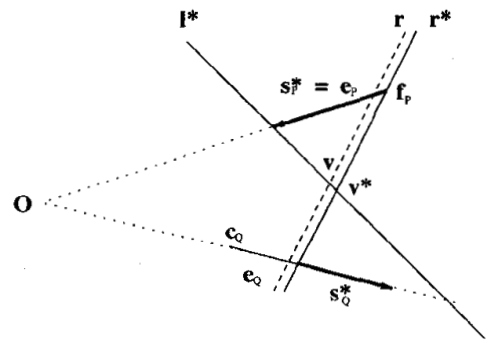

(a)

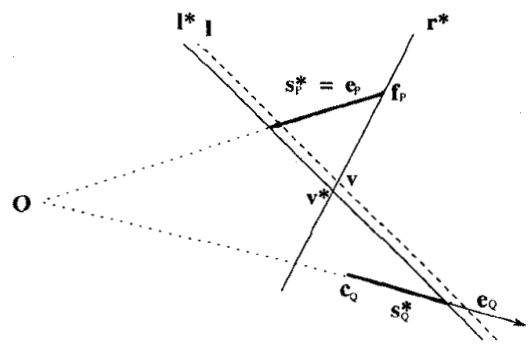

(b)

Fig. 20. Some nonoptimal grasps that can be improved. (a) Case where $s_{P}^{\star} \equiv e_{P}, \beta_{l} \geq d_{Q}$. (b) $s_{P}^{\star} \equiv e_{P}, \beta_{l}<d_{Q}$.

4) otherwise, $d\left(s_{P}^{\star}\right)=d\left(s_{Q}^{\star}\right)$ and either

$$
\begin{aligned}
& \text { a) } \quad \alpha_{r}=0 \text { and } \beta_{l} \leq d_{Q} \quad \text { or } \\
& \text { b) } \beta_{l}=d_{Q} \text { and } \alpha_{r} \geq 0 \text {. }
\end{aligned}
$$

Proof: By Theorem 1, either $\alpha_{r}=0$ or $\beta_{l}=d_{Q}$. The "if" parts of the conditions corresponds to the different situations for which one of these criteria is satisfied, so that each case can be considered separately.

Condition (1) corresponds to the case where both of the edges lie within $C^{\times}\left(\mathrm{v}^{\star}\right)$. Obviously, if $\left(s_{P}^{\star}, s_{Q}^{\star}\right)$ satisfy this condition, it defines an optimal grasp. The converse is also obvious.

Conditions (2) and (3) both correspond to the situation in which one edge is within $C^{\times}\left(\mathbf{v}^{\star}\right)$ but only a portion of the other edge is within $C^{\times}\left(\mathbf{v}^{\star}\right)$. The converse of these two conditions are obvious. We will prove the forward implication for condition (2); the same arguments can be used to prove the case involving condition (3). Condition (2) applies to the situation where $s_{P}^{\star}=e_{P}$ and $s_{Q}^{\star} \neq e_{Q}$.

If $\left(s_{P}^{\star}, s_{Q}^{\star}\right)$ defines can optimal grasp on $e_{P}$ and $e_{Q}$ and $s_{P}^{\star}=e_{P}$, then there must exist a grasp generating cone such that

$$
\alpha_{r}=0, \alpha_{l}=d_{P} \text {. }
$$

Let $C^{\times}=\left\{\mathbf{l}^{\star}, \mathbf{r}^{\star}\right\}$ be a generating cone of $\left(s_{P}^{\star}, s_{Q}^{\star}\right)$, but $d\left(s_{Q}^{\star}\right)<$ $d\left(s_{P}^{\star}\right)$. There are two cases to consider: either $\beta_{l} \geq d_{Q}$ or $\beta_{l}<d_{Q}$. Suppose $\beta_{l} \geq d_{Q}$, as shown in Fig. 20(a). Since $s_{Q}^{\star} \neq e_{Q}$, it follows that $\beta_{r}>0$. Now, we construct the grasp generating cone $C^{\times}=\left\{1^{\star}, \mathbf{r}\right\}$ such that

$$
d\left(s_{P}\right)=d\left(s_{Q}^{\star}\right)+\epsilon
$$

for some $\epsilon>0$, i.e.,

$$
\mathbf{r}=\left(\mathbf{f}_{P}+\left[\alpha_{l}-d\left(s_{Q}^{\star}\right)+\epsilon\right] \mathbf{u}_{P}\right)+\rho \mathbf{u}_{r} .
$$

If $\beta$ denotes the characterizing parameter of the intersection of the line $\mathbf{r}$ with $e_{Q}$, then $\beta_{r}>\beta$. Hence, we have

$$
\begin{aligned}
d\left(s_{Q}\right) & =d\left(s_{Q}^{\star}\right)+\left(\beta_{r}-\operatorname{MAX}\{0, \beta\}\right) \\
& >d\left(s_{Q}^{\star}\right) .
\end{aligned}
$$




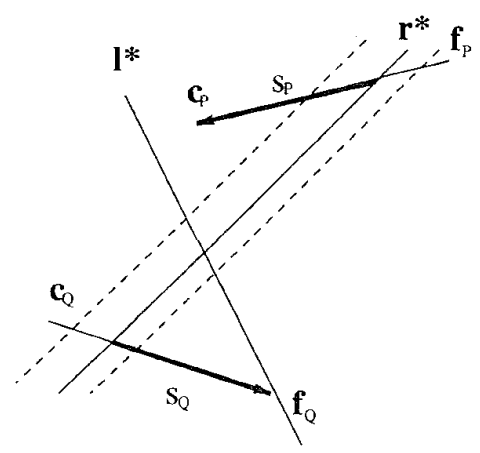

(a)

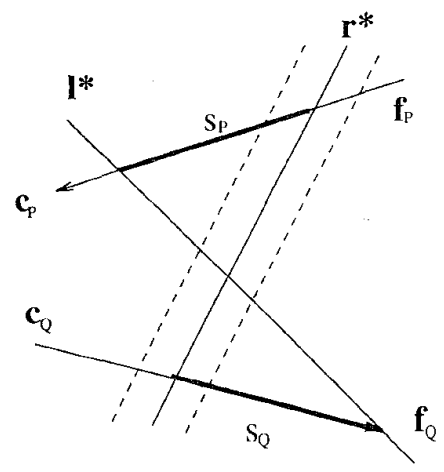

(b)

Fig. 21. Optimal grasps with $\mathbf{f}_{Q}$ lying on $\mathbf{l}^{\star}$.

Thus

$$
\begin{gathered}
\operatorname{MIN}\left\{d\left(s_{P}\right), d\left(s_{Q}\right)\right\}>d\left(s_{Q}^{\star}\right) \\
\Rightarrow \operatorname{MIN}\left\{d\left(s_{P}\right), d\left(s_{Q}\right)\right\}>\operatorname{MIN}\left\{d\left(s_{P}^{\star}\right), d\left(s_{Q}^{\star}\right)\right\} . \\
\Rightarrow \Leftarrow
\end{gathered}
$$

By similar arguments, we can show that if $\beta_{l}<d_{Q}$, then the grasp defined by $\left(s_{P}^{\star}, s_{Q}^{\star}\right)$ is not optimal. A pictorial illustration for this case is given by Fig. 20(b).

Condition (4) corresponds to the case where a portion of each edge lie inside of $C^{\times}\left(\mathbf{v}^{\star}\right)$. The two configurations associated with this case correspond to whether $\mathbf{r}^{\star}$ passes through $\mathbf{f}_{P}$ or $\mathbf{l}^{\star}$ passes through $f_{Q}$ (by Theorem 1). Let's consider the situation for the conditional statement (4b); the same arguments can be used to prove the case for the conditional statement (4a).

$(\Longrightarrow)$ Suppose $d\left(s_{P}^{\star}\right)=d\left(s_{Q}^{\star}\right)$, but there does not exist a grasp generating cone such that the conditional statement $(4 \mathrm{~b})$ is satisfied. Since $s_{P}^{\star} \neq e_{P}$ and $s_{Q}^{\star} \neq e_{Q}$, and $\mathbf{l}^{\star}$ passes through $\mathbf{f}_{Q}$, we have

$$
\beta_{l}=d_{Q} \text { and } \beta_{r}>0 \text {. }
$$

If $\alpha_{r}<0$, then

$$
\mathbf{C}^{\times}(\mathbf{v})=\left\{\mathbf{v}, \mathbf{l}^{\star}, \mathbf{r}=f_{P}+\beta \mu_{r}\right\}
$$

satisfies

$$
\begin{aligned}
& d\left(s_{P}\right)=d\left(s_{P}^{\star}\right) \\
& d\left(s_{Q}\right)>d\left(s_{Q}^{\star}\right)
\end{aligned}
$$

but this contradicts our assumption that the grasp defined by $\left(s_{P}^{\star}, s_{Q}^{\star}\right)$ is optimal.

$\left(\Longleftarrow\right.$ ) Suppose that $\left(s_{P}^{\star}, s_{Q}^{\star}\right)$ satisfies the conditional statement (4b) as depicted by Fig 21 . Now, consider the independent regions of contact, $\left(s_{P}, s_{Q}\right)$, produced by another grasp generating cone of form $C^{\times}=\left\{l^{\star}, \mathbf{r}\right\}$. Denote the intersections of $e_{Q}$ with $\mathbf{r}^{\star}$ and $\mathbf{r}$ as $3_{r}^{*}$ and $\beta_{r}$, respectively. Then

$$
\begin{cases}d\left(s_{P}^{\star}\right)>d\left(s_{P}\right) & \text { if } \beta_{r}<\beta_{r}^{\star} \\ d\left(s_{Q}^{\star}\right)>d\left(s_{Q}\right) & \text { if } \beta_{r}>\beta_{r}^{\star} .\end{cases}
$$

Thus

$$
\operatorname{MIN}\left\{d\left(s_{P^{\prime}}^{\star}\right), d\left(s_{Q}^{\star}\right)\right\} \geq \operatorname{MIN}\left\{d\left(s_{P}\right), d\left(s_{Q}\right)\right\} .
$$

Similarly, the independent regions of contact, $\left(s_{P}, s_{Q}\right)$, generated by any grasp generating cone of the form $C^{\times}=\left\{\mathbf{l}, \mathbf{r}^{\star}\right\}$ satisfy

$$
\operatorname{MIN}\left\{d\left(s_{P}^{*}\right), d\left(s_{Q}^{\star}\right)\right\} \geq \operatorname{MIN}\left\{d\left(s_{P}\right), d\left(s_{Q}\right)\right\} .
$$

If $C^{x}$ is the set of all such grasp generating cones described above, then all other independent regions of contact on $e_{P}$ and $e_{Q}$ can be obtained by translating a cone, $C^{\times}(\mathbf{v}) \in C^{\times}$, along the segment $\overline{\mathrm{Ov}}$ toward $\mathrm{O}$. Therefore, the translation constant is negative. Apply the Translation Lemma, we get

$$
\operatorname{MIN}\left\{d\left(s_{P}^{\star}\right), d\left(s_{Q}^{\star}\right)\right\} \geq \operatorname{MIN}\left\{d\left(s_{P}\right), d\left(s_{Q}\right)\right\}
$$

for all $\left(s_{P}, s_{Q}\right)$ of $e_{P}$ and $e_{Q}$. Hence, the grasp defined by $\left(s_{P}^{\star}, s_{Q}^{\star}\right)$ is optimal.

Q.E.D.

\section{REFERENCES}

[1] T. Lozano-Perez, J. L. Jones, E. Mazer, P. A. O'Donnell, and E. L. Grimson, "Handey: A robot system that recognizes, plans, and manipulates," in Proc. IEEE Int. Conf. Robotics and Automation, 1987, pp. 843-849.

[2] P. Tournassoud and T. Lozano-Perez, "Regrasping," in Proc. IEEE Int. Conf. Robotics and Automation, 1987, pp. 1924-1928.

[3] L. B. Gatrell, "CAD-based grasp synthesis," S.M. thesis, Univ. Utah, Provo, 1988

[4] R. A. Wolter and A. C. Woo, "Automatic generation of gripping positions," IEEE Trans. Syst., Man, Cybern. vol. SMC-15, pp. 204-213, 1985.

[5] J. Barber, R. A. Volz, R. Desai, R. Rubinfeld, B. Schipper, and J. Wolter, "Automatic two-fingered grip selection," in Proc. IEEE Int. Conf. Robotics and Automation, 1986, pp. 890-896.

[6] R. C. Brost, "Automatic grasp planning in the presence of uncertainty," in Proc. IEEE Int. Conf. Robotics and Automation 1986, pp. 1575-1581.

[7] M. Teichmann and B. Mishra, "Reactive algorithms for grasping using a modified parallel jaw gripper," in Proc. IEEE Int. Conf. Robotics and Automation, 1994, pp. 1931-1936.

[8] B. Mishra, J. T. Schwartz, and M. Sharir, "On the existence and synthesis of multifinger positive grips," Algorithmica, pp. 541-558, 1987.

[9] X. Markenscoff, L. Ni, and C. H. Papadimitriou, "The geometry of grasping," Int. J. Robot. Res., pp. 61-74, Feb. 1990.

[10] J. Hong, G. Lafferriere, B. Mishra, and X. Tan, "Fine manipulation with multifinger hands," in Proc. IEEE Int. Conf. Robotics and Automation, pp. $1568-1573,1990$.

[11] T. Omata, "Fingertip positions of a multifingered hand," in Proc. IEEE Int. Conf. Robotics and Automation, 1990, pp. 1562-1567.

[12] Y. C. Park and G. P. Starr, "Grasp synthesis of polygonal objects," in Proc. IEEE Int. Conf. Robotics and Automation, 1990, pp. 1574-1580.

[13] B. Faverjon and J. Ponce, "On computing two-finger force-closure grasps of curved 2D objects," in Proc. IEEE Int. Conf. Robotics and Automation, 1991, pp. 424429.

[14] C. Ferrari and J. Canny, "Planning optimal grasps," in Proc. IEEE Int Conf. Robotics and Automation, pp. 2290-2295.

[15] V. Nguyen, "The synthesis of stable force-closure grasps," S.M. thesis, Mass. Inst. Technol., Cambridge, 1986.

[16] Z. Li and S. S. Sastry, "Task-oriented optimal grasping by multifingered robot hands," IEEE J. Robot. Automat., vol. 4, no. 1, pp. 32-44. Feb. 1988.

[17] D. G. Kirkpatrick, B. Mishra, and C. K. Yap, "Quantitative Steinitz's theorems with applications to multifingered grasping." in Discrete \& Computational Geometry, vol. 7, no. 3. New York: Springer-Verlag, 1992, pp. 295-318. 